\title{
Probing protoplanetary disks with Aperture Masking
}

\author{
S. Lacour ${ }^{1}$, P. Tuthill ${ }^{2}$ and S. Casassus ${ }^{3}$ \\ ${ }^{1}$ LESIA, CNRS/UMR-8109, Observatoire de Paris, UPMC, Université Paris Diderot, 5 place \\ Jules Janssen, F-92195, Meudon, France \\ ${ }^{2}$ School of Physics, University of Sydney, NSW 2006, Australia, \\ ${ }^{3}$ Departamento de Astronomía, Universidad de Chile, Camino del Observatorio 1515, Las \\ Condes, Santiago, Chile
}

\begin{abstract}
The interaction between planetary formation and protostellar disks is among the most critical remaining pieces in the puzzle of solar system assembly. Leading theoretical models are constructed around two distinct scenarios: gravitational instabilities and core accretion. The physics of each applies to quite different epochs of formation, and exhibits complex dependencies on parameters like disk density and viscosity. Untangling the effects such processes have on the final planetary statistics necessitates direct observation of exoplanets in their primordial state, prior to orbital migration. Furthermore, detailed study of the environment, such as the way the planets shape the protostellar disk by driving accretion streams across disk gaps, will also constrain formation models. Aperture masking interferometry has demonstrated a unique ability to probe the gaps within stellar disks. It has twin advantages of a higher dynamic range at the diffraction limit $(\lambda / \mathrm{D})$ than differential imaging, while at the same time giving very extensive UV coverage compared to long baseline interferometry.
\end{abstract}

Keywords. Disks, planet formation, aperture masking, interferometry

Transitional disk are rare, accounting for around $10 \%$ of the T Tauri population. They are believed to form the interface between class II pre-main sequence, and class III systems hosting debris disks. They are characterized by a distinctive dip in their infrared SED which suggests that a partially evacuated gap exists in the inner region of the protoplanetary disk (e.g. Calvet et al. 2002). The profound implications for studies of planetary formation have become increasingly apparent with the confirmation of the diskgap architecture by sub-millimetre measurements (e.g. Andrews et al. 2011) and optical interferometry (e.g. Olofsson et al. 2011). A number of mechanisms have been proposed to cause these gaps, including extensive grain growth (Dullemond \& Dominik 2005), photo evaporation (Clarke et al. 2001) and binarity (Ireland \& Kraus 2008), however most exciting for exoplanetary science is the potential for such systems to arise from the tidal barrier created by dynamical interaction with low-mass disk objects (Bryden et al. 1999; Papaloizou 2007). These different mechanisms can be distinguished by studying the distribution of the gas and dust within the gaps. For example, gravitational clearing by a large (stellar) companion or photoevaporation from the primary would almost completely evacuate the inner regions, while a less massive planetary companion would allow gas and small dust grains to exist within its orbit (Lubow et al. 1999). Furthermore, in the latter case, the measurement of the size and distribution of this material would allow the orbit and mass of the planetary companion to be constrained (Andrews et al. 2011).

Aperture masking on an 8 meter telescope is a key technique to understand the physics and the dynamics of the shaping of such holes in transition disks. This is mainly due to the critical nature of the observational parameter space opened by this method. 


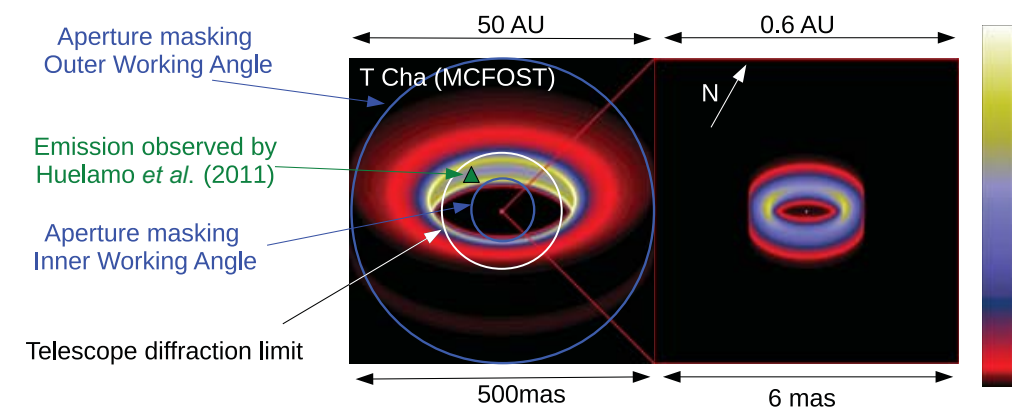

Figure 1. The inner (right) and outer (left) disks from T Cha modeling on which is over-plotted the observational window offered by aperture masking. In the near infrared, on an 8-meter telescope, the inner working angle is 30mas, and the outer working angle around 300mas (at which point differential techniques like ADI become more efficient). At the distance of the Cha I association, it means an IWA at 3AU, and an OWA at $30 \mathrm{AU}$.

Aperture masking is able to provide information on an object at a resolution between $0.5 \lambda / \mathrm{D}$ to a few $\lambda / \mathrm{D}$ with a dynamic range of several hundred (Biller et al. 2012). At the typical distance of young star-forming clouds $(\approx 150 \mathrm{pc})$, aperture masking can probe with high dynamic range the immediate vicinity of disk-enshrouded stars at a resolution of a few AUs. As a result, this technique has yielded the key discovery of the protoplanet LkCa 15 b (Kraus \& Ireland 2012), which explains the gap in this system. Also, SAM detected structure around a compact source in the inner disk surrounding HD142527 (Biller et al. 2012), and yielded resolved observations of the gap in T Cha (Huélamo et al. 2011; Olofsson et al. 2013). Thus SAM has unravelled touchstones systems for testing planet formation models. But discoveries are open to interpretation. Although the clear interferometric signals reported by separate teams are hardly open to challenge, could it be that the interpretation as a sub-stellar companion is too simplistic? The problem is twofold: first, aperture masking delivers data streams which have complex observables difficult to understand and model by teams not profoundly involved in the technique. Second, the data interpretation must be done with as wide a variety of tools and constraints as possible. Incisive science will only result from combination of all available observational data combined with advanced modeling to globally interpret the information from different techniques and wavebands.

\section{References}

Andrews, S. M., Wilner, D. J., Espaillat, C., et al. 2011, ApJ, 732, 42

Biller, B., Lacour, S., Juhász, A., et al. 2012, ApJ, 753, L38

Bryden, G., Chen, X., Lin, D. N. C., Nelson, R. P., \& Papaloizou, J. C. B. 1999, ApJ, 514, 344

Calvet, N., D'Alessio, P., Hartmann, L., et al. 2002, ApJ, 568, 1008

Clarke, C. J., Gendrin, A., \& Sotomayor, M. 2001, MNRAS, 328, 485

Dullemond, C. P. \& Dominik, C. 2005, A\&A, 434, 971

Huélamo, N., Lacour, S., Tuthill, P., et al. 2011, A\&A, 528, L7

Ireland, M. J. \& Kraus, A. L. 2008, ApJ, 678, L59

Kraus, A. L. \& Ireland, M. J. 2012, ApJ, 745, 5

Lubow, S. H., Seibert, M., \& Artymowicz, P. 1999, ApJ, 526, 1001

Olofsson, J., Benisty, M., Augereau, J.-C., et al. 2011, A\&A, 528, L6

Olofsson, J., Benisty, M., Le Bouquin, J.-B., et al. 2013, A\&A, 552, A4

Papaloizou, J. C. B. 2007, A\&A, 463, 775 